Biol. Cybern. 54, 125-132 (1986)

\title{
Constraints for Joint Angle Control of the Human Arm
}

\author{
H. Cruse \\ Department of Biology, University of Bielefeld, D-4800 Bielefeld 1, Federal Republic of Germany
}

\begin{abstract}
The targeting movements of a human arm were examined when restricted to a horizontal plane. The three joints at shoulder, elbow, and wrist are allowed to move. Thus, the system is redundant and needs constraints. A model calculation using a simple form of constraint is found to describe the experimental results: a cost function is applied to each joint. The constraint consists in minimizing the sum of the costs of all three joints. The cost functions might be interpreted as to describing the energy cost necessary to move the joint and/or represent a mechanism which avoids singularities.
\end{abstract}

\section{Introduction}

In both technical and biological systems there exist manipulators which are able to reach a given object within their workspace in order to handle it for a given task. Highly developed examples are robot manipulators (see Luh 1983, for a summary) and the human arm. In the first case it is interesting to develop laws for the control of the movement of the manipulator. In the second case the already existing control mechanisms could be of interest. This paper deals with the latter problem.

In order to move the tip of the manipulator, the endeffector, in a given workspace, the manipulator consists of links which are connected by joints. The usual robot manipulator has exactly as many joints as needed to reach the object with its endeffector. In contrast, biological manipulators as well as recent developments of artificial manipulators may have additional joints to bring the endeffector to a given position. Such systems have more degrees of freedom than necessary because for a given position of the endeffector an infinite number of possibilities exist for the configuration of the links and joints of the manipulator. These systems possess redundancy. To be able to chose a definite combination of joint variables (the joint angles in the case of revolute joints) a redundant systems needs additional constraints. These constraints effectively decrease the degree of freedom of such a primarily "underconstrained" system. In this paper the control of movement of the human arm is investigated. Three joints, shoulder, elbow, and wrist, were allowed to move, but only in the horizontal plane which contained the shoulder joint. The palm had to be held vertically. Thus, three revolute joints could be used whose axes of rotation were perpendicular to the horizontal plane. The position of the tip of the endeffector, in this case a pointer attached to the palm, is determined by two cartesian coordinates in the horizontal plane. Therefore, two joints were sufficient to move the endeffector to any point in the workspace. The existence of the third joint produced an additional degree of freedom and therefore made the system redundant. The objective of this paper is to find the nature of the contsraints in this 3-linked human arm system.

\section{Methods}

The experiments were performed with 4 subjects (one female and three males) aged between 23 and 43 years with no physical abnormalities or disabilities. A rigid pointer was mounted at the palm of the right hand so that the effective length of the wrist (wrist joint to end of pointer) was about the same as that of the forearm. The subject was seated in front of a table, high enough to constraint the arm to move in a horizontal plane on the surface of the table. During the whole session the shoulder was fixed by being pressed against a vertical metal pole mounted on the edge of the table. Three starting points $(A, B$, and $C)$ and 20 target points were 




Fig. 1. Top view of the experimental arrangement. The 20 target points are placed on a horizontal plane in front of the subject. The target points 18,19 , and 20 appear twice, above and below the number. Usually the upper points are used except for one subject, the results of which are shown in Fig. 3. In this case the lower points are used. Two extreme arm positions are shown for target point 6 . These are given by the geometrical limitations. The three measured angles are marked

marked on the table (Fig. 1) and could be seen by the subject. The subject was asked to move the tip of the pointer from each of the three starting points to each of the 20 target points. Thus a total of 60 movements were performed.

Before the session the axes of rotation of the three joints were marked by black dots glued to the skin. The positions of these dots were observed using a video camera viewing the table from above and a monitor whose screen was covered by a transparent millimeter grid. After the point reached the target point, the coordinate values of the joints were read from the monitor and processed by computer in order to calculate the angle values.

The angles were defined as shown for two examples in Fig. 1. The shoulder angle was defined to be zero when the upper arm was positioned on the line connecting both shoulder joints. Movement in the anterior direction is positive. Due to the limited exactness of measurement and of slight movement of the skin carrying the dots the angles could be measured within a range of $\pm 2^{\circ}$.

Before the experiment began the subject was allowed to perform a series of movements between all target points to become familiar with the situation. During the experiment the subject was asked to make the movements in a "comfortable" way. In particular there was no special request concerning the speed of the movement.
In all subjects the movements were performed in the same temporal series, starting with the starting point $A$ to target point 1, then from $B$ to 1 and from $C$ to 1 . This procedure was repeated for all target points continuing with target point 2,3 etc. up to point 20 . This was permissible because preliminary experiments showed that the order of the target points does not influence the results.

This method of measurement is very simple. However, it only allowed measurement of the static situation at the end of the movement, not the dynamic part, i.e. the angles assumed during the movement. This will be the subject of a following paper.

\section{Results}

\section{Geometrical Constraints}

When considering the constraints of a manipulator, two different sorts of constraints have to be distinguished: a) control constraints which, as described above, are only due to redundant manipulator and decrease the degree of freedom and b) geometrical constraints which are due to both redundant and nonredundant manipulators. The geometrical constraints are those which determine the boundary of the workspace. They are given by the geometrical properties of the manipulator, i.e. the lengths of its links and the extreme angle values which can be adopted by the different joints. Figure 1 as an example shows the two extreme positions of the arm of a given subject for the target point 6 . This figure shows that for a given target point and given link lengths each angle can only adopt values within a definite range. As these ranges depend upon the position of the target point, Table 1 gives the ranges of the three angles for each target point for one subject as an example. It can be seen that in spite of the geometrical constraints there is still a considerable range of possible movement for each joint. However, in this subject the target positions 19 and 20 are obviously at the boundary of the workspace: the ranges of possible movements are in the order of the exactness of measurement. This means that in this case the experimental results are completely determined by the geometry of the system.

Whereas Fig. 1 shows two extreme positions in the workspace (cartesian) coordinates and Table 1 gives the ranges of the possible joint angles, Fig. 2 demonstrates for three selected examples (target points 4, 9 , and 12) the geometrically possible joint values in joint space coordinates. The values of the three joint angles are presented here in two diagrams, each using the elbow angle as abscissa and the wrist and shoulder angle as ordinate, respectively. This diagram is used lateron to show the experimental data. 
Table 1. The maximum ranges for the three angles at wrist (above), elbow (middle), and shoulder (below) derived as if only geometrical constraints existed. The values are given for each target point in degrees. They are taken from the subject whose data are shown in Fig. 4

\begin{tabular}{|c|c|c|c|c|c|c|}
\hline & & & 92 & 80 & 72 & \\
\hline & & 1. & 75 & 2. 62 & 3. 56 & \\
\hline & & & 24 & 17 & 14 & \\
\hline 90 & 105 & & 105 & 99 & 98 & 79 \\
\hline 4. 66 & 5. 83 & 6. & 79 & 7. 76 & 8.78 & 9. 60 \\
\hline 22 & 34 & & 31 & 88 & 26 & 16 \\
\hline 99 & 73 & & 44 & 29 & 35 & 64 \\
\hline 10.77 & 11. 63 & 12. & 41 & 13. 27 & 14. 35 & 15. 60 \\
\hline 38 & 44 & & 33 & 19 & 19 & 25 \\
\hline 65 & 30 & & 19 & 5 & 7 & \\
\hline 16. 59 & 17. 24 & 18. & 19 & 19. 4 & 20. 8 & \\
\hline 49 & 49 & & 20 & 4 & 4 & \\
\hline
\end{tabular}


Fig. 2. Three examples of joint angle ranges. The joint angles which were possible if only geometrical constraints were existing are shown for the target points 4,9 , and 12 . The values are shown in joint space coordinates: abscissa is elbow angle, ordinate is wrist angle in the upper diagram, and shoulder angle in the lower diagram

\section{Experimental Data}

As will be shown the results obtained from the four subjects differed considerably. Therefore it was not possible to calculate mean values for all data and the results are presented for each subject separately.

For each of the 20 target points three measurements were performed as each target point was approached from one of the three starting points $A, B$, and $C$. Mean values were calculated from these three measurements and are presented for the four subjects in Figs. 3-6 in the joint space coordinates as described above. There appears to be an obvious difference between the subjects. The strategies which are used by the four subjects might represent four different states of a continuum and the results are arranged to follow an intuitive order: When moving from Figs. 3-6, the wrist angle seems to be held more and more rigid at approximately $180-190^{\circ}$ unless the geometrical conditions constraint the system to move also the wrist joint. One feature should be mentioned which seems to be common to all subjects although the overall results
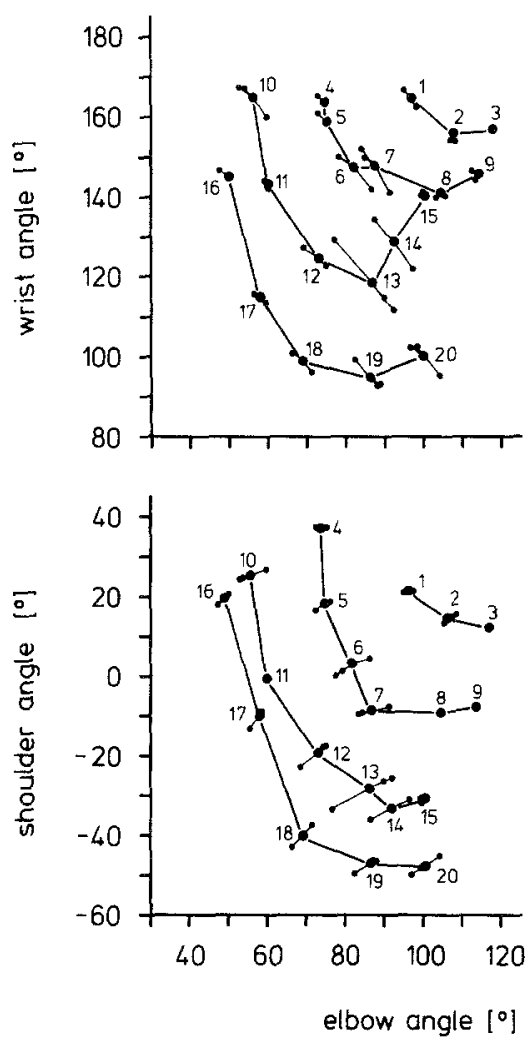

Fig. 3. Results for subject (a). The data are presented in joint space coordinates as explained for Fig. 2. The mean values are given as closed circles and are numbered according to the corresponding target point. The mean value of those target points which lie on horizontal rows in Fig. 1 are connected by thick lines. The three small dots connected by thin lines with the mean values show the individual values obtained by approaching every target point from each of the three starting points 



Fig. 4. Results for subject (b). The data are presented in joint space coordinates as explained for Fig. 2. The mean values are given as closed circles and are numbered according to the corresponding target point. The mean values of those target points which lie on horizontal rows in Fig. 1 are connected by thick lines. The small dots connected by a thin line with the corresponding mean value represent the results obtained by the model calculation

are different. The data of Figs. 3, 5, and 6 show a sudden increase of wrist angle for target 15. In Fig. 4 this is true for target 20 instead. As this subject had smaller link lengths target point 20 in this subject might correspond to target point 15 of the other subjects.

For one subject, not only the mean values but also the individual measurement values are shown in Fig. 3. As the deviations are about the same in all four subjects, for the sake of clarity the individual values are omitted in the other figures. The standard deviations collected from all subjects were $\pm 1.2^{\circ}$ for the shoulder angle, $\pm 2.4^{\circ}$ for the elbow angle, and $\pm 3.8^{\circ}$ for the wrist angle. The results show that the deviations are much smaller than the possible range due to the geometrical constraints (see Fig. 2). In one subject the experiment was repeated after three weeks but with a different order (i.e. beginning with target point 20 ) and no significantly different results were found.
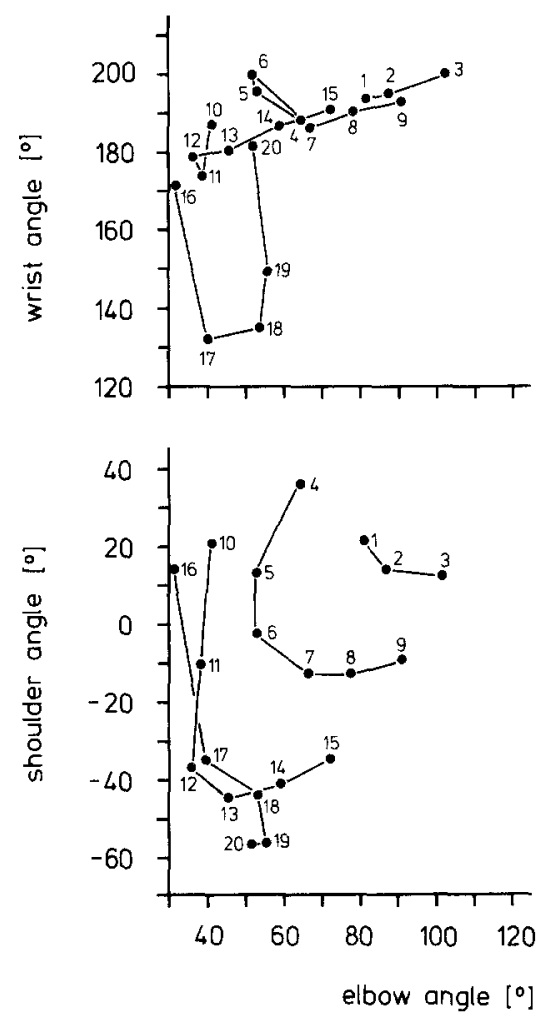

Fig. 5. Results for subject (c). The data are presented in joint space coordinates as explained for Fig. 2 . The mean values are given as closed circles and are numbered according to the corresponding target point. The mean values of those target points which lie on horizontal rows in Fig. 1 are connected by thick lines

Do these final joint angles depend on the direction by which the target point is approached? To clarify this question the deviations of all individual measurements from their mean values were summarized for all four subjects but were separated by refering to their starting point being either $A, B$ or $C$. The frequencies of these deviations are shown in Fig. 7a-c, respectively. Only wrist angles were considered here because they showed the largest deviations. The results show that there is indeed a significant difference between the results of $A$ and $C$ (U-test, $p \ll 0.1 \%$ ). However, the absolute values of the deviations were very small $\left(A\right.$ : median: $-2^{\circ} ; B$ : $\left.0^{\circ} ; C:-1^{\circ}\right)$.

\section{Model Data}

The problem addressed in this paper deals with the question of whether it is possible to find rules which form constraints for the redundant system under view. In the literature concerning the control of robot manipulators several simple methods have been previously proposed (see Discussion). However, it might well be the case that in the biological system relatively complicated strategies are used. The obvious dif- 

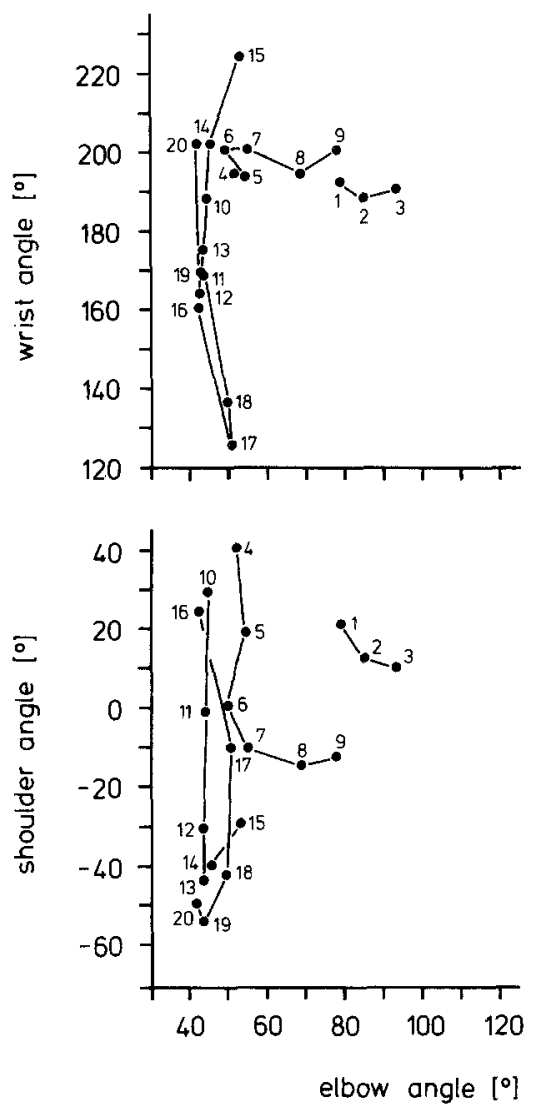

Fig. 6. Results for subject (d). The data are presented in joint space coordinates as explained for Fig. 2. The mean values are given as closed circles and are numbered according to the corresponding target point. The mean values of those target points which lie on horizontal rows in Fig. 1 are connected by thick lines

ferences between the subjects might for example suggest that the individual apply very different strategies. Or it might be that one individual uses different strategies for target points lying in different regions of the workspace.

A simple method to constraint a redundant robot manipulator has been proposed by several authors (for summary see Hollerbach and Suh 1985; Liegois 1977); a similar method was applied to the control of the body position of a six-legged animal walking over uneven surfaces (Cruse 1976). It is assumed that a cost function is attached to each joint. Moving a joint away from a minimum cost position in any direction increases the cost of this joint. The costs of all three joints are summed up to give the total cost value. The position with the minimum total cost value is be chosen. (For possible interpretations of such a cost function see Discussion.)

This model which introduces a constraint by assuming a minimum total cost condition was applied to the data. For this purpose the form of the three cost

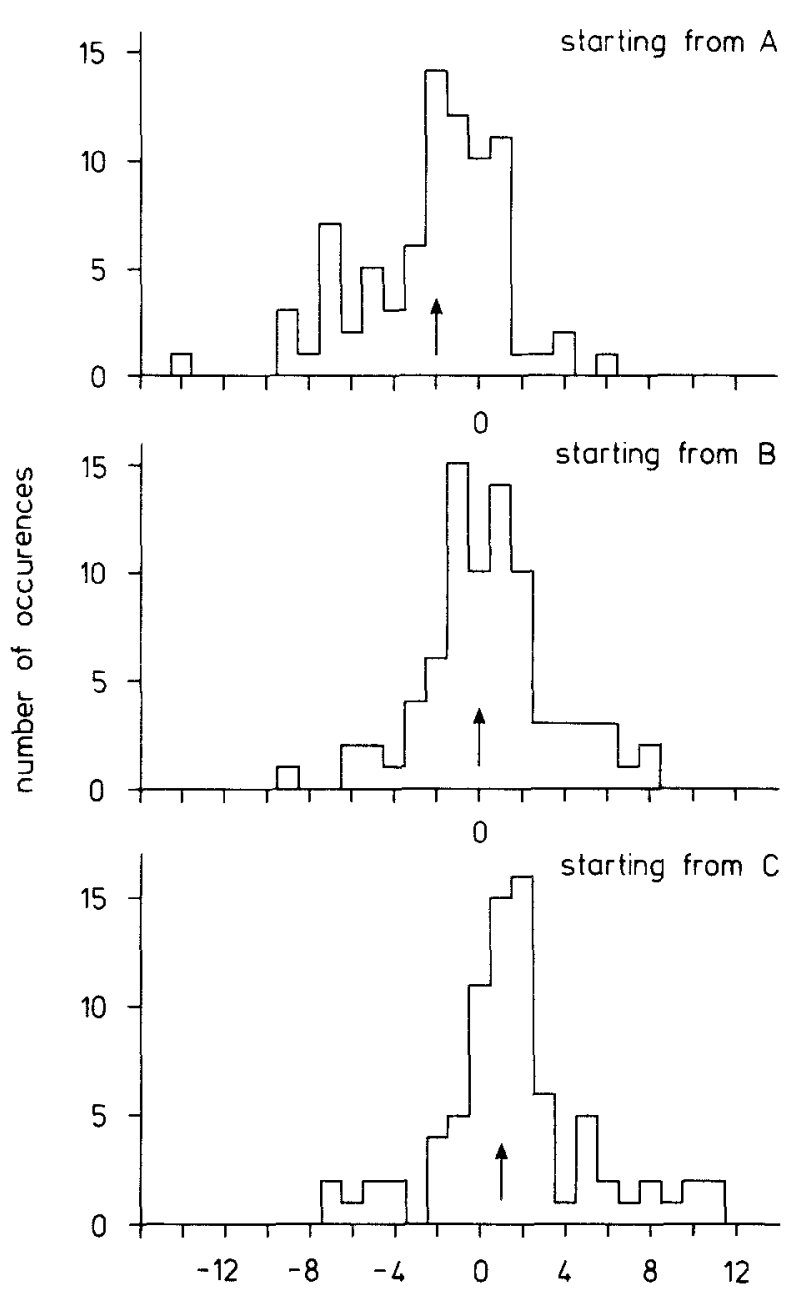

deviation of actual wrist angle from mean value $\left[^{\circ}\right]$

Fig. 7. The deviation of wrist angles from the mean value. The diagram shows the difference between the individual value of the wrist angle and the mean value obtained from the three measurements when the target point is approached from each of the three starting points $A, B$, and $C$. (a) when starting from $A$; (b) when starting from $B$; (c) when starting from $C$

functions (shoulder, elbow, wrist) were varied in order to find out whether a set of cost functions could be found which produced arm positions similar to those found in the experiments. The cost functions applied consisted of the sum of two exponential functions with different sign for the argument. Eventually the steepness was increased by adding an additional exponential function or a linear function onto it. The morphological joint limits were fitted to the function by an additional strong increase in the cost.

The deviation between the model and the experimental data of all subjects is shown in Fig. 8 for each angle. The standard deviations are $\pm 2.6^{\circ}$ for the shoulder angle, $\pm 3.6^{\circ}$ for the elbow angle, and $\pm 6.3^{\circ}$ for the wrist angle. These results were obtained by 

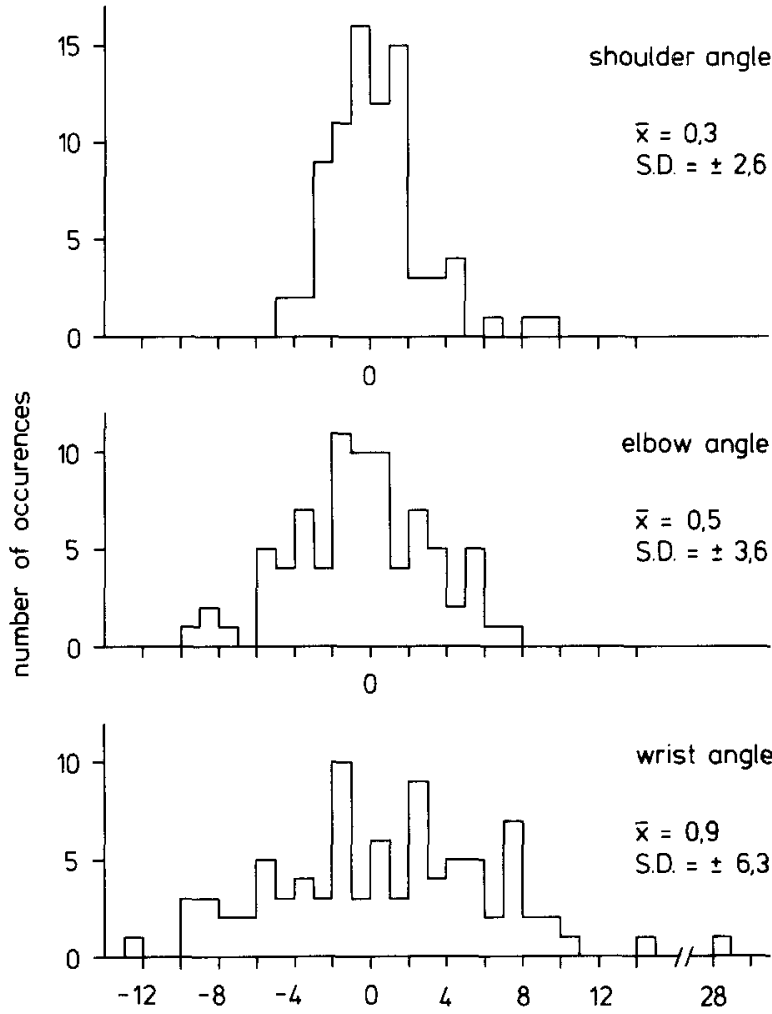

difference between experimental dato and model results $\left[{ }^{\circ}\right]$

Fig. 8. The differences between model and experimental data. The differences were collected for all four subjects and shown separately for the three joint angles: (a) shoulder, (b) elbow, and (c) wrist joint angle

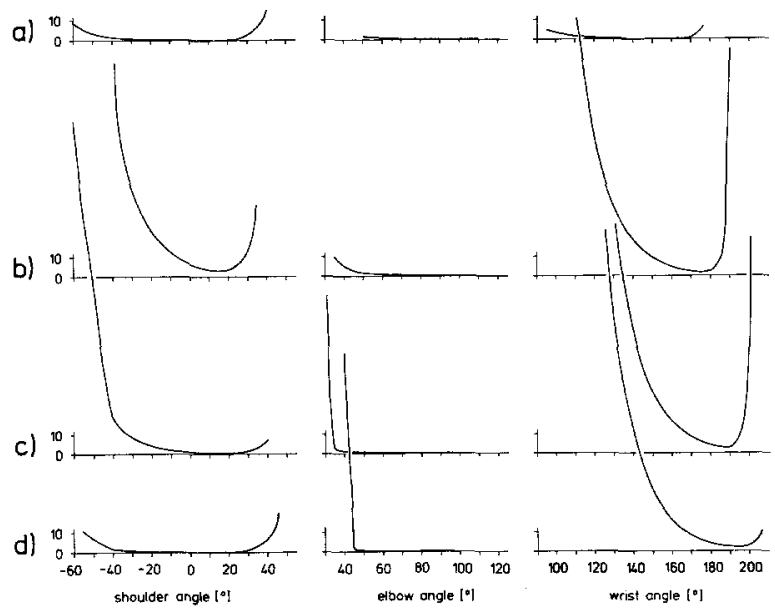

Fig. 9a-d. The cost functions for shoulder, elbow, and wrist joint as assumed for the model calculation. a-d show the four sets of cost functions applied for the four subjects whose data are given in Figs. 3-6, respectively. The ordinates describe the cost value in relative units

using the cost functions presented in Fig. 9. The deviation between model and experimental data was nearly twice the standard deviation of the experimental data which were $1.2^{\circ}, 2.4^{\circ}$, and $3.8^{\circ}$, respectively. For visual inspection of the deviation between experi- mental results and model data, the latter are also shown for one subject in Fig. 4. It might well be possible that a more sophisticated approximation process would provide a better fit to the data: the shape of the cost functions were changed intuitively according to visual inspection of the deviations obtained by the former calculation. In particular, there seems to be one effect which is only qualitatively described by the model calculation. This is the strong increase of the wrist angle at the target position 15, mentioned earlier. Particularly for the data shown in Fig. 6 this position shows a large deviation. Although it cannot be ruled out that a further effort to change the shape of the cost functions would improve the model, another possibility has to be taken into account. It might well be that the cost function of a joint is not simply a function of the angle of this joint, but may depend, at least to some extent, also on the position of the other joints. Such a model was indeed found to improve the results, but was not considered further because the minor improvements did not justify the increases in complexity. Such a change of the model will not be introduced until experiments in progress provide further information as to whether the cost functions are dependent on other joint angles. Thus, at the present time the model given is considered to provide a sufficient first order description of the data.

\section{Discussion}

The control of a redundant manipulator requires more computational costs compared to a non-redundant manipulator. Nevertheless, many biological and some robot manipulators are constructed to have redundancy. Redundancy provides several advantages (see Yoshikawa 1985; Hollerbach and Suh 1985; Salisbury and Abramowitz 1985): First, redundancy can help to avoid obstacles. In a non-redundant manipulator an obstacle in the workspace might prevent the manipulator from moving the endeffector along a specific path. With additional joints such obstacles could be avoided without perturbing the path of the endeffector. Second, redundancy can help to avoid so called singularities. Singularities are positions in the workspace where the possible directions of movements of the endeffector are limited. This means that the degree of freedom is decreased for such positions.

This is immediately obvious for singularities, which appear at the outer boundary of the workspace. This outer boundary cannot be changed by additional joints. However, "inner" boundaries may also occur, for example, with a two-link manipulator whose links have different lengths. In this case the endeffector cannot reach into a circle around the central joint. This type of singularity or those produced by limited joint 
ranges could be avoided by additional joints. Second, additional joints can increase the workspace. Third, redundant systems permit the optimization of torques around the different joints (Hollerbach 1985). If one joint, e.g. the wrist joint can produce only a small torque compared to the other joints, then an alignment of the wrist with the vector of an external force acting on the endeffector would minimize the torque developed by the wrist joint. Only a redundant system would allow fulfillment of this additional condition. Thus, redundant manipulators are in general superior to non-redundant ones and it is of interest how the movement of a biological redundant manipulator is controlled, i.e. what sort of constraints are developed.

The experimental results presented in Fig. 7 show that in our paradigm, as a first order approximation, the joint angles at the end of the movement do not depend on the direction of the movement and are only controlled by the position of the target point. This result rules out a possible strategy described by Korein (1985), the so called "reach hierarchy" algorithm. Following this proposal to solve the problem of redundancy, first only the proximal link, i.e. the upper arm should move as long as the target gets in the workspace of the subsystem "forearm and hand," than the forearm moves until the target is in the workspace of the hand and finally the hand moves to the target. Using this strategy the final joint angle would depend on the position of the starting point. The same was true when applying the strategy that the total amount of energy spent for the movement should be minimized. Neither strategy was used to define the principal rules for the control of arm movement in our paradigm.

The results are well described if we assume that a cost function exists for each joint. The additional constraint necessary for the control of joint position in the redundant system is given by the condition that the combination of angles is chosen which produces the minimum total cost. Two possible, not necessarily exclusive, interpretations of these cost functions will be mentioned. First, the cost function might describe the physiological cost, i.e. the amount of energy necessary to hold the joint in a given position. The sense of applying the minimum cost constraint would then be to minimize muscle energy. Second, the cost function might be completely independent of the actual physiological costs but might represent a function defined within the nervous system. In this case the sense of the application of the cost function might be to keep the joints away from extreme angles near the joint limits. This avoids the singularities described above. Further experiments must be performed to distinguish between these two possibilities.

Finally, I wish to consider how the three angle values which give the minimum total cost might be determined. One possible method corresponds to that used in this model calculation: a three dimensional space is constructed using the three angles (shoulder, elbow, wrist) as coordinates. Every point in that space corresponds to one arm position. To each of these points a total cost value is assigned. When a target point is given, a path in this three-dimensional space can be calculated which corresponds to all arm positions which allow the arm to reach the target point (see Fig. 2). Then the minimum for all cost values of the points lying on this path has to be calculated in order to obtain the actual joint angle values. These three values might then be used as commands to the individual joints.

This method, intuitively, seems unlikely for the human brain because much computation would be necessary. However, the calculation of the three joint angle values need not be repeated for each movement. As an alternative, one could also imagine that the angle values for each target point were precalculated (for example during a learning process) and were stored in a look-up table. Although this would simplify the calculation process, it would require much storage space. Particularly, if one remembers that the real arm can move in a three-dimensional space and has more degrees of freedom than in our paradigm (where rotation about the long axis of the upper arm and of the fore arm as well as vertical movements of the upper arm are excluded, not counting the hand joints). In the real situation the required amount of storage would be much higher. This is even more important when the wrist is prolonged by a pointer as in our experiment. Each different length would require a totally new set of look-up tables. Thus, one is inclined to postulate other mechanisms as being more likely.

Possibly the real method does not calculate merely the final angle values. These values might simply result from the determination of the varying angles adopted during the movement, values which were completely neglected in this paper. Future investigations of the dynamical behaviour of the arm should provide more insight into the way in which the final angles are determined.

Acknowledgements. This work was supported by the Deutsche Forschungsgemeinschaft (Cr 58/5-1). It was performed in the laboratory of Prof. Dr. L. Stark, UC Berkeley, to whome I want to express my warm thanks for his invitation and cordial support. I also want to thank to Dr. D. Graham for proof reading the English manuscript.

\section{References}

Cruse H (1976) The control of body position in the stick insect (Carausius morosus) when walking over uneven surfaces. Biol Cybern 24:25-33 
Hollerbach JM (1985) Optimum kinematic design for a seven degree of freedom manipulator. In: Hanafusa $H$, Inoue $H$ (eds) Robotoc research. The 2nd Int. Symp. of Robotics Research, MIT Press, Cambridge, Mass. London pp 215-222

Hollerbach JM, Suh KC (1985) Redundancy resolution of manipulators through torque optimization. Proceed. of the 1985 IEEE International Conference on Robotics and Automation. IEEE Computer Soc Press, Silver Spring, Md

Korein JU (1985) A geometric investigation of reach. MIT Press, Cambridge, Mass

Liegois A (1977) Automatic supervisory control of the configuration and behaviour of multibody mechanisms. IEEE Trans Syst Man Cybern 7:868-871

Luh JYS (1983) Conventional controller design for industrial robots - a tutorial. IEEE Trans Syst Man Cybern 13:298-315
Salisbury JK, Abramowitz JD (1985) Design and control of a redundant mechanism for small motion. Proceed. of the 1985 IEEE International Conference on Robotics and Automation. IEEE Computer Soc Press, Silver Spring, Md Yoshikawa T (1985) Manipulability and redundancy of robotic mechanism. Proceed. of the 1985 IEEE International Conference of Robotics and Automation. IEEE Computer Soc Press, Silver Spring, Md

Received: December 24, 1985

Prof. Dr. H. Cruse

Fakultät für Biologie

Universität Bielefeld

Postfach 8640

D-4800 Bielefeld 1

Federal Republic of Germany 